\title{
Effect of respiratory training apparatus and vibration expectoration vest on expectoration in patients with lung cancer resection
}

Original article

Xiao-Jing Guo ${ }^{a}$, Li-Li Wej ${ }^{b, *}$, Xin-Hui Li ${ }^{a}$, Ning- Ning Yu ${ }^{a}$, Shao-Bo Gao ${ }^{c}$, Dong-Yan Qinn ${ }^{d}$, Fu-Mei Chua, Kun $\mathrm{Li}^{\text {a }}$

${ }^{a}$ Cardiovascular Surgery, Affiliated Hospital of Qingdao University, Qingdao, Shandong 266000, China

${ }^{b}$ Nursing Department, Affiliated Hospital of Qingdao University, Qingdao, Shandong 266000, China

'Obstetrics Department, Affiliated Hospital of Qingdao University, Qingdao, Shandong 266000, China

${ }^{\circ}$ Gynecology Department, Affiliated Hospital of Qingdao University, Qingdao, Shandong 266000, China

Received: 20 October 2017; Accepted: 20 November 2017; Published: 20 March 2018

Abstract: Objective: The aim of this study was to explore the safe and effective method of expectoration in the preoperative period of patients with lung cancer resection and to promote the rehabilitation of patients.

Methods: A total of 100 cases of lung cancer patients undergoing elective surgery were divided into the observation group and the control group, with 50 cases in each group. The control group was treated with vibration expectoration vest for expectoration during the perioperative period, and the observation group was treated with respiratory function exerciser that has expectoration function in the perioperative period, three times a day, and the effect was evaluated after 5 days.

Results: The number of patients in the observation group after the first expectoration time was significantly less than that of the contro group $(P<0.001)$. Pain score, pulmonary atelectasis, and pulmonary infection rate of the observation group were significantly lower than those of the control group; the hospitalization time was significantly shorter than that of the control group; and the difference was statistically significant $(P<0.05)$.

Conclusions: Lobectomy for lung cancer patients with perioperative respiratory training for respiratory function exercise, compared with conventional methods, is helpful for postoperative expectoration and to reduce the incidence of adverse events.

Keywords: lung cancer • sputum • pain • lung carcinoma • physiatry • lung function • nursing intervention

(c) Shanxi Medical Periodical Press.

\section{Introduction}

Patients undergoing pulmonary lobectomy for tracheal catheterization under general anesthesia and endotracheal intubation may cause physical stimulation to the airway, airway secretion induced by. ${ }^{1}$ Because of postoperative wound pain, the patients dare not force cough, leading to sputum accumulation in the respiratory tract, which easily leads to respiratory complications. ${ }^{2}$ At

* Corresponding author.

E-mail: 13573828157@163.com (L.-L.Wei).

2 Open Access. ๑ 2018 Xiao-Jing Guo et al., published by Sciendo. (c) BY-NC-ND This work is licensed under the Creative Commons Attribution NonCommercial-NoDerivatives 4.0 License. present, the main sputum expectoration methods in the clinical operation include percussion back, acupressure stimulation, mechanical vibration expectoration vest, and respiratory training device with expectoration function as the phlegm elimination program for interventional intervention. In order to explore the advantageous expectoration scheme, the author tried to compare the effect of the sputum elimination program with the respiratory training device and the vibration expectoration vest as the representative of the vibration expectoration program, so as to provide references for clinical nursing 
and related research. The study was approved by the independent ethics committee of the hospital. The subjects gave informed consent and volunteered to participate in this study.

\section{Data and methods}

\subsection{General information}

In this cross-sectional study, performed in the Department of Thoracic Surgery of a tertiary academic hospital, 100 lung cancer patients undergoing thoracoscopic lobectomy from November 2016 to May 2017 were included. Patients were divided into the observation group and the control group, with 50 cases in each group. Inclusion criteria are as follows: according to the Chinese Society of Respiratory Diseases Diagnosis, patients how were diagnosed with lung cancer and the patients with surgical conditions; no obvious serious basic diseases in liver, kidney, and other important organs; pulmonary function tests; the preoperative no ventilation disorder; and should not have the mental and the central nervous system symptoms. Exclusion criteria are as follows: (1) patients who received chemoradiotherapy within 6 months prior to pulmonary surgery; (2) withdrawal of the patients due to various reasons; and (3) severe illness during the experiment; unable to continue to accept the intervention. The selected 100 cases were divided into two groups according to admission time: 50 cases were treated with vibration expectoration vest, and 50 cases were treated with respiratory training apparatus.

\subsection{Methods}

\subsubsection{Intervention method}

\subsubsection{Basic nursing}

Patients of both the groups received routine nursing in Department of Thoracic Surgery. The following were done before treatment: nurses in charge did the skin preparation for patients; informed the precautions of surgery and basic process during and after the operation in detail to the patients; patients with habits of smoking and drinking alcohol should strictly stop smoking and alcohol; prevention of upper respiratory tract infection; prescribed antibiotics; psychological assessment, and emotional counseling. After the operation, the vital signs of the patients were observed closely; the drainage tube was unobstructed; the supine position was strengthened; and the basic nursing was strengthened so as to avoid complications such as pressure sores and urinary tract infection.

\subsubsection{Sputum scheme}

In this study, perioperative time was defined from preoperative day 2 to day 3 after operation, two groups of patients were inhaled in conventional atomization and drug-resistant effects on the treatment of phlegm, sputum treatment proceed in 1-2 hours after meals. The patients were explained before treatment to get their coordination. After inhalation of atomized liquid for 15-20 minutes, the patients were asked to sit or be in the semirecumbent position. The control group used vibration expectoration vest (model: ZPT-205B, Thales Electronics Co., Ltd.) as a tool for expectoration. According to the patient's body shape, the appropriate vest type was chosen, and after the power supply, the automatic common mode was chosen and monitored carefully to observe the patients' response and vital signs during the treatment, and according to the patients' condition and tolerance ability the intensity of treatment was adjusted to alleviate the patients' discomfort. The treatment time was 15 minutes. The observation group used the breath training device (model: HXQ1512; Jiangsu Deweilan Medical Equipment Co., Ltd.) as a tool to carry out sputum. Two days before the operation, the nurses assisted patients sitting or at semi reclining position, guiding the patients as much as possible to take deep breath by the nose inhale, mouth and tongue contain respiratory trainer blow pipe, using both hands to hold the breath training device, while slowly expiratory phase vibration observation of magnetic vibration, vibration frequency should be able to shake the patients feel breath resistance but can be tolerated and throat itching. The nurses should guide the patients to maintain physical and mental relaxation in the training, rest for 1 minute before second time, and comment on the first row of sputum, pointing out inappropriate, give the correct way of operation, guide the patient to fully grasp the respiratory function exercise process, according to the nursing order exercise. The frequency and duration of exercise were equal to those of the control group, the nurses' responsibility was inspection and supervision, to ensure timely exercise.

\subsubsection{The method of evaluation}

The following were evaluated: (1) Recording of the first expectoration after the operation of two groups that is postoperative time (minutes) was recorded. (2) Two cases of atelectasis and pulmonary infection after the beginning of expectoration exercise were recorded. (3) The Prince-Henry pain score for chest and abdominal surgery was used to evaluate the pain degree of patients 24 hours post operation after expectoration training in two groups. (4) Recoding of the length of hospital stay (days) was recorded. 


\subsubsection{Statistical method}

SPSS 21.0 statistical software was used to process data. $t$-test and $\chi^{2}$ test were performed, and the threshold of significance for all comparisons was 0.05 .

\section{Result}

\subsection{General data of the two groups (Table 1)}

\begin{tabular}{|c|c|c|c|c|}
\hline Items & $\begin{array}{l}\text { Observation } \\
(n=50)\end{array}$ & $\begin{array}{l}\text { Control } \\
(n=50)\end{array}$ & Statistics & $P$ \\
\hline \multicolumn{5}{|l|}{ Gender [n (\%)] } \\
\hline Male & $27(54)$ & $21(42)$ & \multirow{2}{*}{$\chi^{2}=1.442$} & \multirow{2}{*}{0.317} \\
\hline Female & $23(46)$ & $29(58)$ & & \\
\hline \multicolumn{5}{|c|}{ Cancer types [n (\%)] } \\
\hline SCLC & $13(26)$ & $18(36)$ & \multirow{2}{*}{$\chi^{2}=1.169$} & \multirow{2}{*}{0.280} \\
\hline NSCLC & $37(74)$ & $32(64)$ & & \\
\hline \multicolumn{5}{|c|}{ Smoking history [n (\%)] } \\
\hline No & $26(52)$ & $21(42)$ & \multirow{2}{*}{$\chi^{2}=1.004$} & \multirow{2}{*}{0.316} \\
\hline Yes & $24(48)$ & $29(58)$ & & \\
\hline Age (years) & $59.30 \pm 7.40$ & $57.56 \pm 11.23$ & $t=0.915$ & 0.362 \\
\hline$B M I\left(k g / m^{2}\right)$ & $23.95 \pm 3.16$ & $23.64 \pm 4.06$ & $t=0.421$ & 0.675 \\
\hline \multicolumn{5}{|c|}{ Preoperative pulmonary function } \\
\hline $\mathrm{FEV}_{1}$ & $2.89 \pm 0.37$ & $2.67 \pm 0.65$ & $t=2.133$ & 0.054 \\
\hline FVC & $3.46 \pm 0.59$ & $3.20 \pm 0.68$ & $t=1.984$ & 0.059 \\
\hline $\begin{array}{l}\text { Preoperative } \\
\mathrm{SpO}_{2}\end{array}$ & $97.66 \pm 0.85$ & $97.66 \pm 1.12$ & $t=0.001$ & 0.990 \\
\hline
\end{tabular}

Table 1. General data of the two groups.

Notes: SCLC, small cell lung cancer; NSCLC, non-small cell lung cancer; BMI, body mass index; FEV1, forced expiratory volume in one second; FVC, forced vital capacity.

\subsection{Comparison of two groups after the first expectoration time, pain score, length of stay and atelectasis, pulmonary infection cases (Table 2).}

\begin{tabular}{lcccc}
\hline \multicolumn{1}{c}{ Items } & $\begin{array}{c}\text { Observation } \\
(n=50)\end{array}$ & $\begin{array}{c}\text { Control } \\
(n=50)\end{array}$ & Statistics & $P$ \\
\hline \hline $\begin{array}{l}\text { The first postoperative } \\
\text { sputum expectoration } \\
\text { time }\end{array}$ & $\begin{array}{c}178.76 \pm \\
73.52\end{array}$ & $\begin{array}{c}408.00 \pm \\
205.99\end{array}$ & $t=7.411$ & $<0.001$ \\
$\begin{array}{l}\text { 24-hour postoperative } \\
\text { pain score }\end{array}$ & $2.00 \pm 0.67$ & $2.38 \pm 0.73$ & $t=2.721$ & 0.008 \\
$\begin{array}{l}\text { Length of stay after } \\
\text { operation }\end{array}$ & $5.02 \pm 1.08$ & $5.54 \pm 1.22$ & $t=2.263$ & 0.026 \\
$\begin{array}{l}\text { Pulmonary atelectasis } \\
{[n(\%)]}\end{array}$ & $2(4)$ & $8(16)$ & $\chi^{2}=4.000$ & 0.046 \\
$\begin{array}{l}\text { Pulmonary infection } \\
{[n(\%)]}\end{array}$ & $4(8)$ & $11(22)$ & $\chi^{2}=3.843$ & 0.045 \\
\hline
\end{tabular}

Table 2. Comparison of two groups after the first expectoration time, pain score, and length of hospital stay.

\section{Discussion}

The most effective treatment for patients with earlystage lung cancer is resection of the tumor. ${ }^{3}$ However, lung surgery can destroy the thoracic organs to some extent, leading to pulmonary inflammatory exudation, weakening of the lung compliance of patients, thereby reducing the lung function of patients. However, the decline in lung function and the function of oxygen will induce cell hypoxia, so as to produce more viscous tracheal secretion and sputum, not easy to cough up, and if no timely treatment is given, it will cause further deterioration and lead to the occurrence of heart failure and respiratory failure and other adverse events. Therefore, to help patients understand the importance of expectoration and grasp the correct and effective method of expectoration in the perioperative period, make them consciously practice the corresponding expectoration program, accelerate the excretion of sputum, and become an important means to improve the effect of postoperative sputum elimination. In this study, the author analyzed the sputum excretion effect and patient experience of two different expectoration methods, in order to accumulate evidence-based data and lay the data support for patients to better deal with surgery.

The results of this study showed that the patients in the observation group have the shorter time of first sputum discharge than the control group, and the difference was statistically significant $(P<0.001)$, suggesting that the perioperative period use by breathing trainer with sputum expectoration function can significantly reduce the first time of postoperative sputum discharge. Its role is to enable additional air into the airway secretions, reaching the bottom, through the vibration to drive the air in the airway, loosen secretions, so as to improve the effectiveness of cough, expectoration, and promote secretions off. ${ }^{4-6}$ Lung surgery cause a great trauma to the tissue, which causes a strong spasm of the bronchus and induces a large number of airway secretions to stay in the trachea, resulting in a decrease in the effective ventilation volume. Breathing trainer as a campaign tool can make the patients achieve power balance between pulmonary ventilation and pulmonary ventilation and then can start the inspiratory-related muscles of patients with all relevant muscles, breath and respiratory muscle coordination movement, so as to improve the tolerance of respiratory muscle activity, the alveolar expansion, improve the individual ventilation/perfusion ratio, reduce hypoxia and the incidence of hyperlipidemia, and promote aerobic metabolism of cells. ${ }^{7-9}$ When the patient's pulmonary function has been improved, it can help patients to gradually transit from the shallow to slow breathing state to the rapid breathing state and avoid the status of airflow accumulation caused by slow 
breathing state, thus promoting secretion discharge. Through the early removal of tracheal secretions, bronchial secretion retention status of patients can be effectively alleviated, peripheral alveolar collapse status has been restored, and they are not prone to atelectasis. The risk of lung complications is also lower. ${ }^{10,11}$ In this study, the incidence of atelectasis and pulmonary infection in the observation group was significantly lower than that in the control group $(P<0.05)$, which also fully confirmed this view.

Some studies have pointed out that postoperative incision pain is also the main reason why the patient cannot forwardly and effectively remove respiratory tract secretions. ${ }^{2}$ Postoperative lung cancer patients due to disease, trauma, infection, nervous system disease, dehydration, activity limitation, as a result of anesthesia for airway mucociliary movement, reduce the thick sputum, cough, weakness, cough reflex weakens lung tissue elasticity, ${ }^{12}$ at the same time, postoperative wound pain can cause skeletal muscle nervous reflex, causing patients fear of deep breathing, respiratory function exercise compliance decreased, ${ }^{13}$ leading to pulmonary insufficiency, pain can also limit the patient's body posture change, the airway secretions cannot be discharged effectively and timely, increase the chance of pulmonary infection. ${ }^{14}$ In order to reduce postoperative pain, postoperative patients of Department of Thoracic Surgery within 12 hours start the routine use of analgesic drugs. In order to avoid the interference of pain score, in this study, the author compares the expectoration training of two groups of patients with postoperative 24-hour pain score, the observation group pain score was significantly lower than that of control group, and the difference was statistically significant $(P<0.05)$.

Upon comparing the patients' prognosis-related indicators of two groups, the results showed that the postoperative hospital stay of the observation group was shorter than the control group $(P<0.05)$; respiratory-related adverse event rates after operation were lower than the control group $(P<0.05, P<0.05)$, which

\section{References}

1. Huang $Y X$, Zhang YW. Effect of perioperative respiratory training and abdominal breathing exercise in patients undergoing resection of lung cancer. $J$ Nurs Sci. 2017;4:29-31 (in Chinese).

2. Zhang YF, Zhang RZ, Fu LP, et al. The relationship between the degree of sputum irritation and the incidence of pneumonia and atelectasis after lung can save the patient's medical costs, reduce the economic burden to relief the mind of patients to deal with the disease, and will help to a good mental state and the overall prognosis of the disease. ${ }^{15,16}$ With the use of breathing trainer as a tool for expectoration training and later treatment of respiratory function training program, it can fully mobilize the motivation of patients' independent practice, so as to strengthen the subjective initiative of learning and achieve the purpose and effect of strengthening training.

\section{Conclusions}

For patients with lung cancer resection, the introduction of sputum breathing function training device as an interventional tool during perioperative period can promote sputum excretion, accelerate the drainage of postoperative sputum, reduce the incidence of postoperative respiratory complications, relieve pain, and promote rehabilitation. In this study, limited manpower resulted in the limitations in design and implementation of this study, which caused some problems, specifically in the following aspects: (1) only the short-term effects of the two groups were recorded and analyzed, and the long-term effects were not followed up, and hence in the follow-up study, the observation time needs to be extended, in order to understand the long-term effect; (2) We should carry out various forms of health education preoperatively, to improve patients' understanding of the importance of respiratory function exercise before and after surgery, and the ongoing quality control in the course of the experiment is to ensure good patient compliance; (3) We did not analyze the gender and age of patients in any group, and the results are more general; and (4) The pulmonary rehabilitation and pulmonary function tests in postoperative patients should pay attention to breathing and tolerance.

\section{Conflicts of interest}

All contributing authors declare no conflicts of interest. cancer surgery. Chin J Pract Nurs. 2014; 30: 55-56 (in Chinese).

3. Li Y, Lin MZ, Zhang FL, Cai JZ. Clinical application of TCM nursing program for lung cancer. J Adv Nurs Nurses. 2016;1:8-10 (in Chinese).

4. Patterson JE, Bradley JM, Hewitt O, Bradbury I, Elborn JS. Airway clearance in bronchiectasis: 
a randomized crossover trial of active cycle of breathing techniques versus Acapella. Respiration. 2005;72:239-242.

5. dos Santos AP, Guimarães RC, de Carvalho EM, Gastaldi AC. Mechanical behaviors of Flutter VRP1, Shaker, and Acapella devices. Respir Care. 2013;58:298-304.

6. McCarren B, Alison JA. Physiological effects of vibration in subjects with cystic fibrosis. Eur Respir J. 2006;27:1204-1209.

7. West K, Wallen M, Follett J. Acapella vs. PEP mask therapy: a randomised trial in children with cystic fibrosis during respiratory exacerbation. Physiother Theory Pract. 2010;26:143-149.

8. Volsko TA, Difiore J, Chatburn RL. Performance comparison of two oscillating positive expiratory pressure devices: Acapella versus Flutter. Respir Care. 2003;48:124-130.

9. Wada JT, Borges-Santos E, Porras DC, et al. Effects of aerobic training combined with respiratory muscle stretching on the functional exercise capacity and thoracoabdominal kinematics in patients with COPD: a randomized and controlled trial. Int J Chron Obstruct Pulmon Dis. 2016;11: 2691-2700.

10. Yu YY, Liu HY. Effect of nursing on respiratory function exercise in coronary artery bypass surgery. 2013;22:2360-2362 (in Chinese).
11. Jiang $A Q$, He CY. Application of respiratory function exercise device in patients undergoing thoracic surgery. J Qilu Nurs. 2008;12:21-22 (in Chinese).

12. Dong M, Li XZ, Wang Lin. Observation of nursing care of two sputum removal methods used in postoperative sputum of elderly patients with esophageal cancer. Chin General Surgery. 2015;7:1195-1196 (in Chinese).

13. Zhu Y, Liu XX, Lu ZH, Cai MH. Psychological consultation on chemotherapy for postoperative patients with lung cancer. Chin Nurs Res. 2008;7:625-626 (in Chinese).

14. Zhi XY, Department of thoracic surgery clinical expert group of Experts Committee of the Ministry of health. In the department of thoracic surgery path consensus of airway management operation period (2012 Edition). J Clin Cardiovasc Surg China Chest. 2013;3:251-255 (in Chinese).

15. Zhou CL, Wen J, Xie CQ. Feedback method to promote respiratory function exercise in patients with pulmonary tuberculosis and chronic obstructive pulmonary disease. Chin Nurs Educ. 2017;5:368-371 (in Chinese).

16. Pu WQ, Liu K, Wang JJ. Nursing experience of chest physiotherapy for patients after cardiothoracic surgery. PLA Nurs J. 2016;17:53-54 (in Chinese).

How to cite this article: Guo X-J, Wei L-L, Li X-H, et al. Effect of respiratory training apparatus and vibration expectoration vest on expectoration in patients with lung cancer resection. Front Nurs. 2018; 4: $285-290$. https://doi.org/10.1515/fon-2018-0042. 